\title{
Brain death, states of impaired consciousness, and physician-assisted death for end-of-life organ donation and transplantation
}

\author{
Joseph L. Verheijde $\cdot$ Mohamed Y. Rady • \\ Joan L. McGregor
}

Published online: 13 May 2009

(c) The Author(s) 2009. This article is published with open access at Springerlink.com

\begin{abstract}
In 1968, the Harvard criteria equated irreversible coma and apnea (i.e., brain death) with human death and later, the Uniform Determination of Death Act was enacted permitting organ procurement from heart-beating donors. Since then, clinical studies have defined a spectrum of states of impaired consciousness in human beings: coma, akinetic mutism (locked-in syndrome), minimally conscious state, vegetative state and brain death. In this article, we argue against the validity of the Harvard criteria for
\end{abstract}

Joseph L. Verheijde, Mohamed Y. Rady and Joan L. McGregor contributed equally to this work.

\section{J. L. Verheijde - M. Y. Rady}

Bioethics, Policy, and Law Program, Arizona State University, 300 East University Drive, Tempe, AZ 85287, USA

e-mail: jverheijde@mayo.edu

\section{J. L. Verheijde}

Department of Biomedical Ethics, Mayo Clinic Hospital, Mayo Clinic Arizona, 5777 East Mayo Boulevard, Phoenix, AZ 85054, USA

\section{J. L. Verheijde}

Department of Physical Medicine and Rehabilitation, Mayo Clinic Hospital, Mayo Clinic Arizona, 5777 East Mayo

Boulevard, Phoenix, AZ 85054, USA

\section{Y. Rady ( $ه)$}

Department of Critical Care Medicine, Mayo Clinic Hospital, Mayo Clinic Arizona, 5777 East Mayo Boulevard, Phoenix, AZ 85054, USA

e-mail: Rady.Mohamed@mayo.edu

\section{J. L. McGregor}

Department of Philosophy, Arizona State University,

300 East University Drive, Tempe, AZ 85287, USA

e-mail: joan.mcgregor@asu.edu equating brain death with human death. (1) Brain death does not disrupt somatic integrative unity and coordinated biological functioning of a living organism. (2) Neurological criteria of human death fail to determine the precise moment of an organism's death when death is established by circulatory criterion in other states of impaired consciousness for organ procurement with non-heart-beating donation protocols. The criterion of circulatory arrest $75 \mathrm{~s}$ to $5 \mathrm{~min}$ is too short for irreversible cessation of whole brain functions and respiration controlled by the brain stem. (3) Brain-based criteria for determining death with a beating heart exclude relevant anthropologic, psychosocial, cultural, and religious aspects of death and dying in society. (4) Clinical guidelines for determining brain death are not consistently validated by the presence of irreversible brain stem ischemic injury or necrosis on autopsy; therefore, they do not completely exclude reversible loss of integrated neurological functions in donors. The questionable reliability and varying compliance with these guidelines among institutions amplify the risk of determining reversible states of impaired consciousness as irreversible brain death. (5) The scientific uncertainty of defining and determining states of impaired consciousness including brain death have been neither disclosed to the general public nor broadly debated by the medical community or by legal and religious scholars. Heart-beating or non-heartbeating organ procurement from patients with impaired consciousness is de facto a concealed practice of physicianassisted death, and therefore, violates both criminal law and the central tenet of medicine not to do harm to patients. Society must decide if physician-assisted death is permissible and desirable to resolve the conflict about procuring organs from patients with impaired consciousness within the context of the perceived need to enhance the supply of transplantable organs. 
Keywords Brain death · Circulatory death · Ethics . Heart-beating organ procurement .

Impaired consciousness .

Non-heart-beating organ procurement .

Neurological criteria of death - Organ transplantation .

Physician-assisted death · Vegetative state

$\begin{array}{ll}\text { Abbreviations } \\ \text { NHBOD } & \text { Non-heart-beating organ donation } \\ \text { PVS } & \text { Persistent vegetative state } \\ \text { UAGA } & \text { Uniform Anatomic Gift Act } \\ \text { UDDA } & \text { Uniform Determination of Death Act } \\ \text { US } & \text { United States }\end{array}$

\section{Introduction}

Human consciousness has two dimensions: arousal or wakefulness (i.e., level of consciousness) and awareness (i.e., content of consciousness) (Zeman 2001). Clinical studies have defined an overlapping spectrum of pathologic states of impaired consciousness (Fig. 1): coma, akinetic mutism (locked-in syndrome), minimally conscious state, vegetative state and brain death (Wijdicks and Cranford 2005). Brain death is one of the states of impaired consciousness which is characterized by irreversible coma and apnea (Zamperetti et al. 2004). As early as 1968, Henry K. Beecher, chair of the Ad Hoc Committee of the Harvard Medical School to Examine the Definition of Brain-Death, believed that organ donation from those who were "hopelessly unconscious" would be beneficial to society (Giacomini 1997). In that same year, the Ad Hoc Committee introduced the definition and the guidelines for determining "brain death." These guidelines are now being referred to as the Harvard criteria (Beecher and Ad Hoc Committee of the Harvard Medical School to Examine the Definition of Brain-Death 1968). This set of criteria singled out a specific state of impaired consciousness, redefined it as brain death, and equated this specific medical condition with human death (Capron 2001). The equation of brain death with death itself was deemed necessary to improve the likelihood of societal acceptance and legalization of heart-beating organ procurement (Giacomini 1997). Since the concept of brain death was enacted into the Uniform Determination of Death Act (UDDA) and brain death was declared as equivalent to human death (National Conference of Commissioners on Uniform State Laws 1981), this act has been a driving force to permit organ procurement in heart-beating donors.

The procurement of cadaveric organs has been a cornerstone of balancing the supply of and demand for transplantable organs for modern transplantation practice and programs in the United States. The medical condition of "brain death" affects a small number of patients in intensive care units in the United States (President's Commission for the Study of Ethical Problems in Medicine and Biomedical and Behavioral Research 1981; Truog 2007). The rate of organ procurement from brain-dead donors has decreased over time because of improved preventive measures, such as the legal mandate in most states in the United States requiring bicyclists and motorcycle

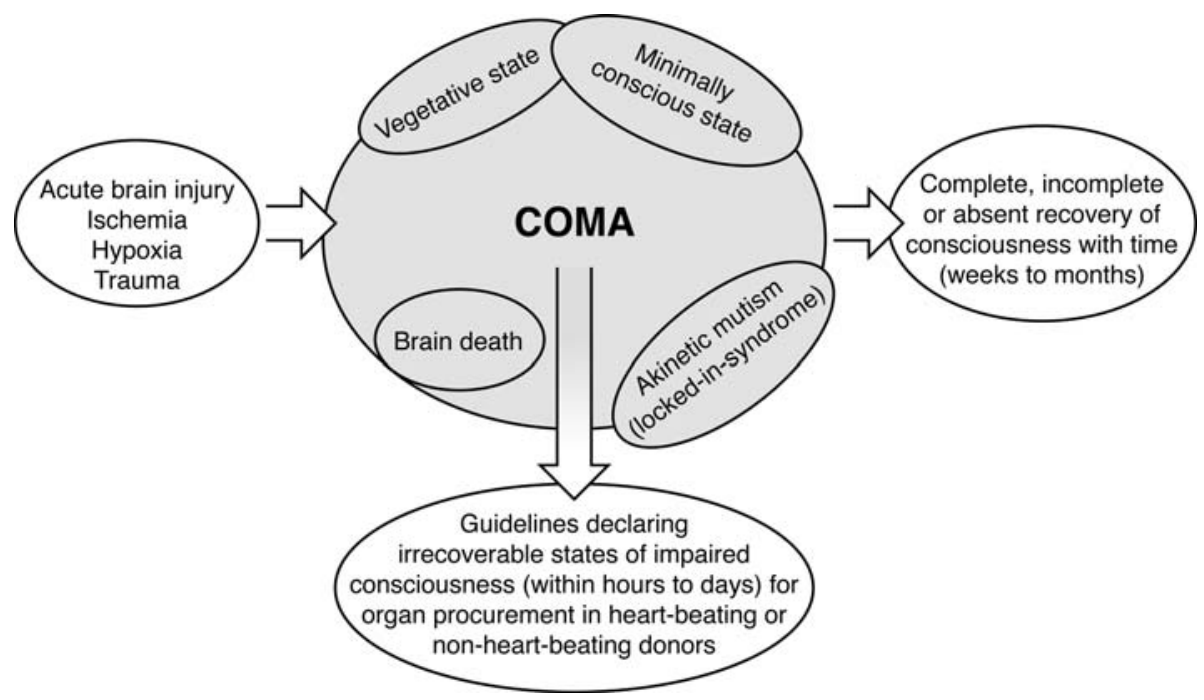

Fig. 1 Overlapping states of impaired consciousness and coma in human beings as sources of transplantable organs. The overlapping spectrum of states of impaired consciousness including coma poses serious diagnostic challenges to clinical practice guidelines attempting to declare irrecoverable conditions in human beings to expedite the procurement of transplantable organs within hours to days of acute brain injury. Errors in determining a potentially reversible coma or a state of impaired consciousness as an unrecoverable condition of brain death can have fatal consequences 
riders over age 18 years to wear helmets, and improved medical care and rehabilitation of the injured human brain. In fact, the reduction of potential brain-dead patients suitable for organ procurement was one of the main reasons to reintroduce non-heart-beating organ donation (NHBOD), also known as donation after cardiac death (DeVita and Snyder 1993). The Pittsburgh protocol, established in 1993, lays the groundwork for the justification and legalization of recovery of transplantable organs from patients with other neurological states of impaired consciousness (Fig. 1) who cannot be declared brain-dead (Hoffenberg et al. 1997). The Pittsburgh protocol defines the loss of arterial pulse with circulatory arrest for $2 \mathrm{~min}$ as the circulatory criterion for determining death in NHBOD. Since the redrafting of the Revised Uniform Anatomical Gift Act (UAGA) in 2006, more patients are likely to be determined to be NHBOD than are those patients determined to be heartbeating donors using the brain death criteria (Verheijde et al. 2007b).

Brain-based criteria for the determination of death, although widely accepted, remain a contentious issue among philosophers, legal scholars, physicians, and other medical professionals. In this review, we reexamine the validity of defining death based exclusively on either neurological or circulatory parameters for organ donation. We contend that neurological or circulatory parameters currently utilized in heart-beating or non-heart-beating procurement of transplantable organs conceal a medical practice of physicianassisted death. The term "physician-assisted death" includes intentional life-ending acts with consent such as active euthanasia or physician-assisted suicide and other intentional life-ending acts performed without explicit request (Quill 2007). This prevailing practice is being performed with no public disclosure ignoring the need for a broad ethical, medical, and legal debate in society.

\section{Brain death as a source of transplantable organs}

\section{Neurological criteria of death}

Historically, the absence of comprehensive criteria for death has led society to rely on a definition based on cardiorespiratory criteria (i.e., the prolonged absence of arterial pulse and respiration), which clearly delineate the line between being alive and being dead.

Technologic advances in life-support systems have made it possible to procure and transplant viable organs from patients who are in irreversible coma and apnea but have retained spontaneous functions of the heart and circulation. In recognition of this reality, a broader definition of death, i.e., brain death, was legislated in the UDDA (President's Commission for the Study of Ethical Problems in Medicine and Biomedical and Behavioral Research 1981). The UDDA explains that a person is determined to be dead upon sustaining either irreversible cessation of circulatory and respiratory functions or irreversible cessation of all brain function, including that of the brain stem (President's Commission for the Study of Ethical Problems in Medicine and Biomedical and Behavioral Research 1981). This determination must be made in accordance with accepted medical standards.

Since human death is a single phenomenon, a definition of death should incorporate contemporary knowledge and an understanding of human biological processes (Fig. 2). Prolonged arrest of blood flow causes irreversible ischemia (and necrosis) of the whole brain and cessation of all integrated neurological functions. From a neurophysiologic perspective, a minimum cerebral perfusion pressure of $15 \mathrm{mmHg}$ is required to sustain blood flow and oxygen supply to the brain (Ivan and with contributions by Melrose 2007). Cerebral perfusion pressure is the gradient between mean arterial and central venous pressures subtracted from the intracranial pressure. The amount of brain necrosis that occurs when the cerebral perfusion pressure falls below this level determines the reversibility or finality of coma in human beings. Absent arterial blood inflow to and venous outflow from the brain on four-vessel cerebral angiography demonstrate complete cessation of intracranial circulation. Prolonged cessation of intracranial circulation eventually results in necrosis and irreversible cessation of integrated neurological functions in heart-beating brain-dead patients.

Although brain-dead patients have no intracranial circulation and (by definition only) irreversible loss of spontaneous respiratory drive and consciousness, the heart and whole body circulation continue to function spontaneously in these patients, i.e. without the use of vasoactive medications for hemodynamic support. Spontaneous circulation maintains many integrated biological functions in braindead patients that are indistinguishable from living human beings, and in some cases these patients can survive on mechanical ventilation for years (Shewmon 1998). Some of the biological functions include wound healing, body temperature regulation, growth to puberty, reproduction, successful completion of pregnancies and delivery of healthy infants (Truog 2007). Therefore, the concept of death based on only neurological criteria-i.e. irreversible cessation of all brain functions or brain death-does not fully encompass the notion of irreversible loss of integrative unity of the organism and its regulatory functions that are essential for life (Joffe 2007b; Karakatsanis and Tsanakas 2002; Shewmon 2001; Zamperetti et al. 2004). In its white paper entitled "Controversies in the determination of death," The President's Council on Bioethics sets out to rebut exactly that position and proposes a new approach to answering the question of whether a diagnosis of "whole 
Fig. 2 Human death is a single phenomenon. Human death is a single phenomenon occurring gradually as a process over time. There is a gradual loss of capacity for somatic integration of the whole body because of an irreversible cessation of all vital and biological functions including circulation, respiration (controlled by the brain stem), and consciousness. The irreversibility of cessation of circulatory and respiratory functions is inter-linked to the onset of whole brain necrosis. Arbitrary neurological criteria and circulatory criterion redefining human death in states of impaired consciousness enable heart-beating and nonheart-beating procurement of transplantable organs, respectively

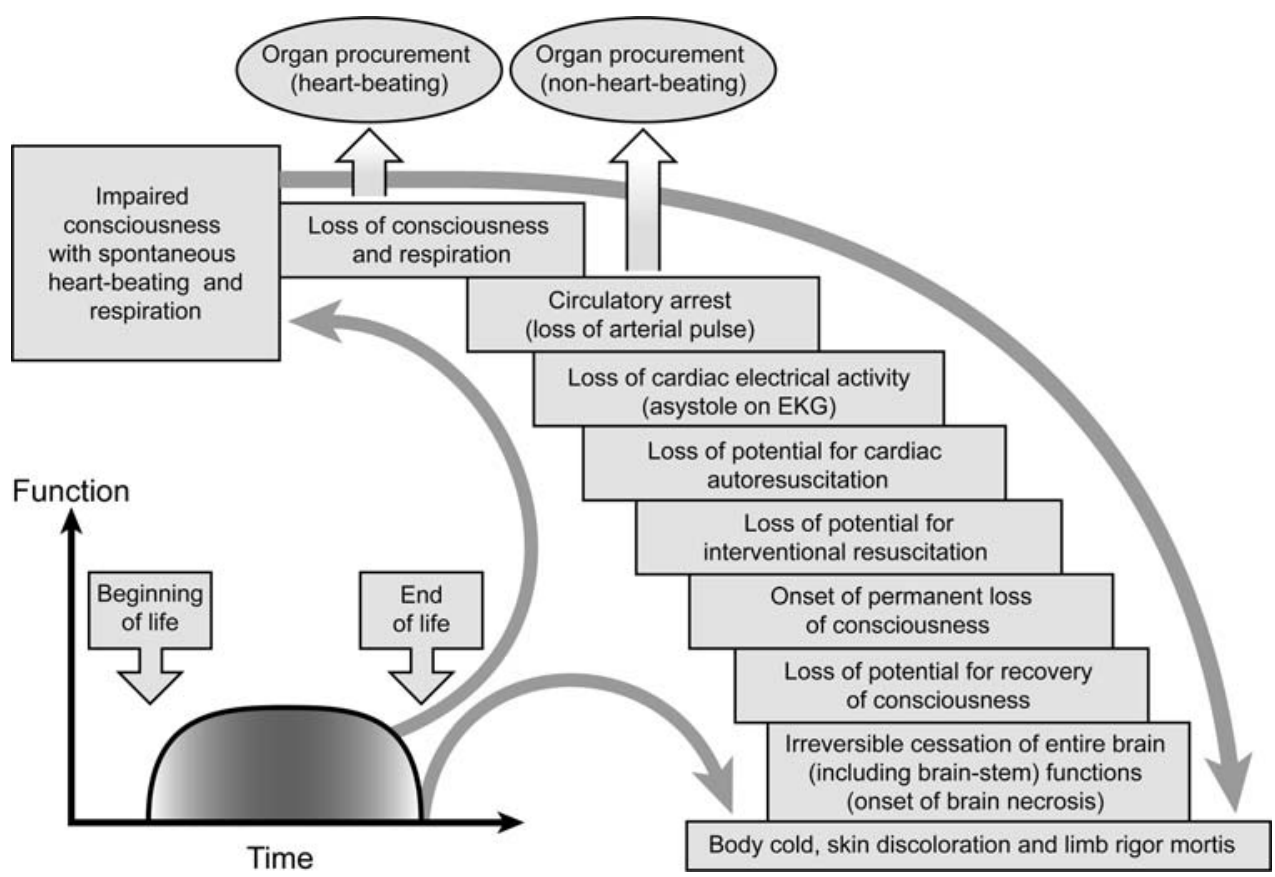

brain death" means that the human being is dead (The President's Council on Bioethics 2008, p. 10). The Council proposes to replace the term "brain death" with the term "total brain failure" for the clinical diagnosis that underlies the current neurological standard (p. 12). The Council recognizes that the central question of whether total brain failure equates with the death of the human being "cannot be settled by appealing exclusively to clinical or pathophysiological facts" (p. 49). In the absence of sufficient empirical evidence for the concept of brain death, to salvage the concept of brain death and to continue support of the current practice of procuring organs from heart-beating donors, the Council proposes to ground this concept in a completely new philosophical rationale. This rationale has not yet been the subject of public debate. It acknowledges that "A human being whose death has been determined according to a neurological standard is the ideal source of transplantable organs" (p. 8). It argues that living organisms preserve themselves and, for that, "must-and can and $d o$-engage in commerce with the surrounding world" (p. 60). "If there are no signs of consciousness and if spontaneous breathing is absent and if the clinical judgment is that these neurophysiological facts cannot be reversed...a once-living patient has now died" (p. 64). The Council's final conclusion is that based on this new rationale the current neurological standard for declaring death is still defensible. However, some Council members have expressed dissent (personal statements published in the white paper pp. 95-100 and pp. 107-119) on the proposed philosophical rationale equating total brain failure (brain death) with human death. In a separate commentary,
Shewmon challenges the validity of the critical role of spontaneous breathing in defining living organisms (Shewmon 2009). The inner drive to breathe is absent not only in total-brain failure patients, but also in conscious patients with lower brain stem lesions and during sleep in patients with Ondine's curse. Therefore, brain death as a state of impaired consciousness and apnea continues to be challenged as a valid concept of human death because of continued insufficient scientific evidence and a less than convincing philosophical rationale. Instead, as critics have postulated, the moment when a living organism is dead, and hence no longer alive is, conceptually, the moment when there is an irreversible cessation of integrative unity of the whole living organism (Byrne and Weaver 2004; Joffe 2007a; Maruya et al. 2008).

Defining death by neurological criteria has additional conceptual implications. The reduction of any definition of death to exclusively neurological terms ignores the anthropologic, cultural, and religious dimensions that many people value highly. The Committee on Increasing Rates of Organ Donation recognizes the role that cultural and religious beliefs play in consenting to organ donation (Committee on Increasing Rates of Organ Donation-Board on Health Sciences Policy-Institute of Medicine 2006). Cultural and religious traditions and beliefs about the treatment of the dead body, beliefs about life after death, and fears of mutilation can also influence decisions about organ donation (p. 35). Policies and practices for procurement of organs must be compatible with conditions deeply rooted in cultural, religious, and legal traditions (p. 4). These traditions, however, greatly vary among global 
communities and pluralistic societies. Reduction of the definition of death to brain-based criteria ignores that the concept of death is not simply bioethical or biomedical in nature but fundamentally shaped and driven by a series of important sociologic influences (Kellehear 2008). Even if there is medical consensus on brain-based criteria and determination of brain death, this consensus is insufficient to conclude that "brain death" is in fact death (Joffe 2007b). To the contrary, for many health care professionals and the general public, the concept of brain death is becoming increasingly abstract and socially disconnected from the nature of death (Kellehear 2008). This paradoxical death, a brain-based determination of death with the physical image of a normally functioning body, creates emotional and cognitive conflicts for many health care professionals and family members (Long et al. 2008).

At the November 9, 2007, meeting of the President's Council on Bioethics, Shewmon (The President's Council on Bioethics 2007b) pointed to the growing consensus that the neurological standard for determining death has become insufficient to appropriately and comprehensively explain brain death:

What has actually happened in the history of this topic is [that] in 1968 we start with the practice. Then there is a revision of statutory laws. Then there is an attempt to come up with diagnostic standards. Then there is a scramble to find rationales for the statutory laws, and there is incoherence and lack of consensus about why destruction or total brain failure, whatever you want to call it, should be death. And so the actual history of brain death has followed exactly the opposite sequence of events that ought to characterize an ideal major socio-legal medical change. So I think at this point in time it's going to be very hard to change how transplantation is done because it's already so ingrained. (The President's Council on Bioethics 2007b)

Accuracy of determining the state of "brain death"

The concept of brain death has been defined as an irreversible state of impaired consciousness diagnosed by universally approved criteria. However, reaching consensus on the "moment of death" can be time consuming. Proponents also point out, what makes the determination of death accurate is the accuracy of following the rules: the practice guidelines that professional associations established for determining brain death (Ivan and with contributions by Melrose 2007). In 1995, The American Academy of Neurology published clinical guidelines for the clinical determination of brain death. These clinical guidelines include: (1) demonstration of coma; (2) evidence of the cause of coma; (3) absence of confounding factors, including hypothermia, the presence of drugs, or electrolyte or endocrine disturbances; (4) absence of brain stem reflexes; (5) absent motor responses; (6) apnea; (7) a repeat evaluation in 6-72 $\mathrm{h}$, with the time based on the patient's age; and (8) confirmatory tests only when specific components of the clinical testing cannot be reliably evaluated (The Quality Standards Subcommittee of the American Academy of Neurology 1995). However, over the past decade, critics have increasingly scrutinized the scientific validity of these clinical guidelines for demonstrating that all brain functions have ceased irreversibly (Joffe 2007b, 2009; Karakatsanis 2008; Karakatsanis and Tsanakas 2002; Shewmon 1997).

The clinical guidelines established for brain death determination and accepted as the medical standard (The Quality Standards Subcommittee of the American Academy of Neurology 1995; Wijdicks 2001) ought to confirm the irreversible cessation of all functions of the entire brain, including the brain stem, before organ donation (President's Commission for the Study of Ethical Problems in Medicine and Biomedical and Behavioral Research 1981); they do not do so. The 'accepted medical standard' must determine with clinical certainty that the brain injury has reached the endpoint of a process of self-perpetuating destruction of neural tissue (The President's Council on Bioethics 2008, p. 38). Clinical and histopathologic observations support that there are serious flaws in the clinical criteria used to declare brain death for heart-beating organ donation. Several critical brain structures remain viable and continue integrated neurological functioning after clinically determined brain death. These include, among others, electroencephalogram activity, brain stem auditory and/or somatosensory evoked potentials and hypothalamic functions (Joffe 2009). First, clinical observations suggest that heart-beating organ donors have residual brain functions, including hormonal and neural responses to nociception and pain during the procurement process (Joffe 2007b). Surgical procurement, which is performed on donors without general anesthesia (Keep 2000), induces hemodynamic responses in donors that are similar to the responses of living organisms in distress (Young and Matta 2000). Second, histopathologic observations in one study suggest that, even when the clinical guidelines of brain death determination are applied appropriately, more than $60 \%$ of heart-beating donors have no or minimal structural disruption of the brain stem at autopsy (Wijdicks and Pfeifer 2008); the absence of neuropathologic features of ischemia or necrosis can also suggest reversibility of ceased brain stem functions. In this particular study, most of these donors were young patients who were declared brain dead by clinical examination and within $24 \mathrm{~h}$ of blunt-force traumatic brain injury (Wijdicks and Pfeifer 2008). 
Reliance upon clinical examination to demonstrate irreversible loss of all brain functions is problematic for the following reasons: (1) the short interval between the initial acute brain injury and clinical declaration of death cannot exclude reversible neurological findings that are masquerading as brain death; (2) no confirmatory test is mandated to validate the complete absence of perfusion pressure and cessation of blood flow to the whole brain; and (3) brain stem ischemic changes, which are structural surrogates of irreversibility, are not always present on autopsy (President's Commission for the Study of Ethical Problems in Medicine and Biomedical and Behavioral Research 1981). The likelihood of incorrect declaration of brain death increases when the timeline necessary for determining irreversible cessation of critical brain functions is shortened because of early organ procurement. Transplant advocates have recommended against performing confirmatory tests when declaring brain death in order to avoid a delay or deferral of organ donation (Greer et al. 2008b). Not performing confirmatory tests may also lead to catastrophic errors in the clinical determination of brain death. The absence of neuropathologic findings of profound brain stem ischemia can suggest reversible causes of coma or perhaps retained neurological activity undetected by clinical examination (Walker 1978). In a Canadian survey, $46 \%$ of neurosurgeons considered the absence of moderate structural damage in both the brain stem and cerebral cortex on brain autopsy to be incompatible with a clinical determination of brain death (Joffe et al. 2007). More than one-third of the neurosurgeons surveyed also considered that some blood flow to the brain or a brain stem with minimal microscopic damage is incompatible with a clinical determination of brain death. Accepting questionable clinical guidelines as the medical standard for early declaration of brain death and organ donation can have fatal consequences when patients whose condition may be salvageable, i.e., amenable to treatment, are determined to be brain dead (Lifesitenews.com 2008; Morales 2008; The President's Council on Bioethics 2008).

It is commonly claimed that, despite unresolved and debated issues about clinical determination of brain death, both the concept and the practice of declaring brain death for organ donation and transplantation have gained wide acceptance in most parts of the world. The development of and adherence to practice guidelines to determine brain death are believed to have elicited universal compliance (Ivan and with contributions by Melrose 2007). Recently, the inconsistency of the medical standard for the clinical determination of brain death for organ donation has been highlighted at leading US neurological hospitals (Laureys and Fins 2008). This inconsistency has opened the door not only for loosening the accepted medical standard for the determination of brain death, but also for potentially sacrificing neurologically salvageable individuals for the sole purpose of organ procurement. In a study by Mathur et al. (2008), organs were procured from 142 pediatric patients who were heart-beating donors, based on braindeath declaration, between January 2000 and December 2004 in southern California. The authors reported their study findings, which had been based on the medicolegal standard: "If it's not documented, it wasn't done." One of 294 neurological examinations documented completion of all the elements required in clinical brain-death examination, $26 \%$ had the apnea test correctly performed, $15 \%$ had at least two examinations performed at the recommended time intervals, and $58 \%$ had cerebral angiography as a confirmatory test. Therefore, in this study, a significant proportion of donors may have been incorrectly declared as brain dead. Similar observations have been noted among adult donors as well; more than two-thirds of leading US neurological hospitals vary widely in their compliance with clinical guidelines for determining brain death before organ donation (Greer et al. 2008a).

\section{Other states of impaired consciousness as sources of transplantable organs}

\section{Circulatory criterion of death}

With the growing need for transplantable organs far exceeding the number of organs procured from brain-dead donors, an alternative criterion to declare death based on cessation of circulation was incorporated in the Pittsburgh NHBOD protocol (DeVita and Snyder 1993). The circulatory criterion to declare death can be used for procuring transplantable organs from patients with other clinical states of impaired consciousness but who cannot be declared clinically brain dead (Fig. 1). Immediately after the introduction of the NHBOD protocol, the criterion of 2 min of circulatory arrest for declaring cardiorespiratory death and commencing organ procurement became controversial (Lynn 1993). Circulatory arrest is determined by the loss of arterial pulse. The Institute of Medicine published a report on the practice and protocols of NHBOD in the United States (Committee on Non-Heart-Beating Transplantation II-The Scientific, Ethical Basis for Practice and Protocols-Division of Health Care Services-Institute of Medicine 2000). Over $90 \%$ of NHBOD protocols in the United States allow for organs to be procured at 5 min or shorter time periods following circulatory arrest (Howard M. Nathan-Medscape Transplantation 2005). Recently, Denver Children's Hospital amended the NHBOD protocol to allow surgical procurement of hearts for transplantation after 75 s of circulatory arrest (Boucek et al. 2008). 
Ongoing debates indicate that not only the scientific validity of the circulatory criterion is questionable (Joffe 2007a) but also that brain-based criteria of death contribute little, if anything, to defining the exact moment of death in NHBOD (Shemie 2007). In human beings, a coronary perfusion pressure of $15 \mathrm{mmHg}$ is sufficient for continued viability of the heart muscle and return of spontaneous circulation after initial circulatory arrest (Paradis et al. 1990). Coronary perfusion pressure is the gradient between asystolic (diastolic) arterial and central venous pressure subtracted from the intrapericardial pressure. Autoresuscitation i.e. spontaneous return of circulation and recovery of heart and brain functions (also called the Lazarus phenomenon) has been reported after $10 \mathrm{~min}$ of circulatory arrest in human beings (Adhiyaman et al. 2007; Joffe 2007a). The presence of coronary and cerebral perfusion pressures after circulatory arrest can explain the Lazarus phenomenon and autoresuscitation in some of the reported cases. This observation is relevant not only because coronary and cerebral perfusion pressures are related to systemic blood flow, but it is also independently controlled by selective arterial and venous vasomotor tones (vascular smooth muscles contraction) and intrapericardial and intracranial pressures surrounding the heart and brain, respectively (Rady et al. 2007). In spite of this physiologic phenomenon, the Institute of Medicine has relied on the loss of systemic arterial pulse and circulation for 2-5 min as the exclusive circulatory criterion for determining death in NHBOD and with no requirement for the complete absence of coronary and cerebral perfusion pressures (Committee on Increasing Rates of Organ Donation-Board on Health Sciences Policy-Institute of Medicine 2006). Neuropathologic features of ischemia or necrosis of the whole brain (and brain stem) become integral observations to establish, with clinical certainty, irreversible apnea and unconsciousness when determining death by the circulatory criterion in NHBOD. The President's Commission for the Study of Ethical Problems in Medicine and Biomedical and Behavioral Research has recommended that circulatory arrest time should be longer than $15 \mathrm{~min}$ for the onset of brain ischemia and to the point that irreversible cessation of brain functions (including respiratory function of the brain stem) is certain (President's Commission for the Study of Ethical Problems in Medicine and Biomedical and Behavioral Research 1981). The criterion of a circulatory arrest time between $75 \mathrm{~s}$ and $5 \mathrm{~min}$ is unlikely to result in irreversible cessation of all integrated neurological functions and respiratory drive in NHBOD. Extracorporeal circulation and cardiopulmonary bypass initiated in NHBOD after 5 min of circulatory arrest invariably reanimate donors during surgical procurement of organs (Dejohn and Zwischenberger 2006). From a legal perspective on determining death with cardiorespiratory criteria, the irreversibility of cessation of circulatory function is interlinked with the irreversibility of cessation of respiratory function (of the brain stem) (National Conference of Commissioners on Uniform State Laws 1981). This point must be emphasized because the UDDA considers human death as a single phenomenon whether determined by neurological criteria or by cardiorespiratory criteria.

Considering death to be the total cessation of life processes characteristic of living organisms throws the practice of applying either circulatory or neurological criteria for declaring death for organ donation into question (Fig. 2). The exact moment when loss of circulation and loss of somatic integration occur is not known and, therefore, the concept of brain death adds little, if any, relevant information about how to determine the precise moment of death in human beings.

Accuracy of determining other states of impaired consciousness

Other states of impaired consciousness, besides brain death, include coma, akinetic mutism (locked-in syndrome), minimally conscious state, and vegetative state. Since 1997, pressure has been growing to expand the recovery of transplantable organs from patients with other states of impaired consciousness, such as those in a vegetative state (Hoffenberg et al. 1997). In September 2007, The President's Council on Bioethics discussed the Draft White Paper on neurological determination of death and renewed the interest in organ procurement from vegetative state to meet an increasing demand for transplantable organs (The President's Council on Bioethics 2007a). The renaming of the neurological standard for determining human death from "brain death" to "total brain failure" preempted the Council's discussion expanding the recovery of transplantable organs from other states of impaired consciousness. In August 2008, a perspective roundtable discussion of organ procurement for transplantation echoed similar interest to abandon traditional neurological criteria for determining death (The New England Journal of Medicine (online) 2008; Truog and Miller 2008).

As highlighted in previous sections of this paper, several shortfalls exist regarding the scientific validity of the concept of brain death. The unassailability of the clinical guidelines for the determination of not only brain death but also that of vegetative state has also been called into question. The American Academy of Neurology has described vegetative state with the following criteria: (1) no evidence of awareness of self or environment and an inability to interact with others; (2) no evidence of sustained, reproducible, purposeful, or voluntary behavioral responses to visual, auditory, tactile, or noxious stimuli; (3) no evidence of language comprehension or expression; (4) 
intermittent wakefulness manifested by the presence of sleep-wake cycles; (5) sufficiently preserved hypothalamic and brain stem autonomic functions to permit survival with medical and nursing care; (6) bowel and bladder incontinence; and (7) variably preserved cranial nerves (pupillary, oculocephalic, corneal, vestibulo-ocular, gag) and spinal reflexes (Report of the Quality Standards Subcommittee of the American Academy of Neurology 1995). The term persistent vegetative state (PVS) is applied when the clinical criteria of vegetative state persist for at least 1 month after the patient has suffered impaired consciousness. The term permanent vegetative state is applied when the clinical criteria of vegetative state persist at least 3 months after nontraumatic brain injury and 12 months after traumatic brain injury in adults and children. Both brain death and PVS are possible outcomes of the comatose state, but, in PVS, the brain stem, which sustains functions such as respiration and circulation, has been spared injury. The American Medical Association has posited that medical treatment, such as artificial nutrition and hydration, may be withdrawn in patients who are in a PVS (Council on Scientific Affairs, Council on Ethical, Judicial Affairs of the American Medical Association 1990). Because these patients may be allowed to die, they may be considered for organ donation; however, unless physician-assisted death is legalized, patients in PVS are ineligible to become organ donors (Detry et al. 2008). Although some have advocated the acceptance of the concept of higher brain death so that organs from patients in permanent vegetative state can be used for transplantation (Hoffenberg et al. 1997), this concept of death has not been endorsed, accepted, or legalized in the United States.

It is often postulated that patients in PVS are devoid of conscious content and cognitive and affective functions and that they have no behavioral evidence of awareness of their external environment (Ivan and with contributions by Melrose 2007). A neurophysiologic explanation of impaired consciousness in PVS underpins this premise, although scientific observations and theory may not be capable of providing a complete account of consciousness (Zeman 2001). Critics have countered, however, that the evidence from human brain imaging studies as well as neurological damage in animals and humans suggests that some form of consciousness can survive the brain damage that commonly causes vegetative state (Panksepp et al. 2007). Neuroscientific evidence indicates that raw emotional or affective feelings (primary-process affects) can exist without cognitive awareness of those feelings. The fact that patients in a PVS can have the capacity to experience affective feelings in the absence of any reflective awareness represents a diametrically opposite position to the one claiming that these patients are devoid of conscious content and cognitive and affective functions. Many aspects of human cognition can go on in the absence of reflective awareness. Functional neuroimaging methods have demonstrated that aspects of speech perception, emotional processing, language comprehension, and even conscious awareness might be retained in some patients who behaviorally meet all of the criteria that define PVS (Owen and Coleman 2008). The diagnosis of PVS is made primarily based on clinical judgment and without performing static and functional neuroimaging and electrodiagnostic studies to confirm this diagnosis with certainty (Wijdicks and Cranford 2005). When diagnosing vegetative state, clinicians may not be as meticulous in their thought processes as they should be, particularly because the need for transplantable organs has risen to the level of what has been called a national health crisis. The risk of misdiagnosing another reversible condition as vegetative state is greater because the vegetative state is not as common as are other prolonged states of impaired consciousness. Shewmon has cautioned that the very use of the term vegetative state itself predisposes physicians to sloppy thinking and employing logical fallacies by implying that something is 'by definition' what is actually an intrinsically unverifiable hypothesis (Shewmon 2004). The clinical criteria for the diagnosis of vegetative state cannot be proven to be valid beyond doubt. The clinical criteria for the diagnosis of vegetative state are a challenge to the premise that good medical research involves, among other aspects, "hypotheses susceptible of proof and disproof, and a methodology that systematically rules out all other explanations" (Shewmon 2004). The accurate determination of PVS requires, among other criteria, clear and robust standards that can be followed in a consistent fashion by the medical community (Rifkinson-Mann 2003). Currently, there is neither consensus on the criteria that encompass the spectrum of PVS nor agreement on the criteria that distinguish this diagnosis from other states of impaired consciousness (Cusack et al. 2000). One retrospective study looked at the clinical records of 40 patients who had received a diagnosis of being in a vegetative state; 17 patients $(43 \%)$ were found to be in other states of impaired consciousness, i.e., had a misdiagnosis of being in a vegetative state (Andrews et al. 1996). All of these open-ended questions and uncertainties surrounding the diagnosis of PVS (The House of Representatives of the 144th General Assembly of the State of Delaware 2008), as with brain-dead, indeed make it medically, ethically, and legally impossible to procure organs by following heart-beating procurement procedures in patients who are in a vegetative state. After a careful analysis of various viewpoints, the President's Council on Bioethics has rejected the concept of higher brain or neocortical failure alone (with normal brain stem functions) for determining human death and procuring transplantable organs (The President's Council on Bioethics 2008). 
The scientific validity and accuracy of clinical guidelines declaring other states of impaired consciousness as unrecoverable within a few days after non-traumatic brain injury have also been called into question. The American Academy of Neurology formulated practice parameters for predicting unrecoverable coma within 3 days of acute brain injury after successful cardiopulmonary resuscitation (Wijdicks et al. 2006). The practice parameters were derived from retrospective analysis of published studies over a time period of 40 years. The predictive accuracy of the American Academy of Neurology practice parameters have not been prospectively validated in either multicenter or multinational studies. Nevertheless, the practice parameters and clinical guidelines declaring states of impaired consciousness or coma unrecoverable within days have become essential prerequisites to facilitate early recovery of transplantable organs (Fig. 1).

\section{Religion and physician-assisted death in organ procurement}

It is commonly stated that all religions approve of organ donation (Ivan and with contributions by Melrose 2007; Woien et al. 2006); however, some states in the United States allow religious beliefs to take precedence over the concept of brain death (Capron 2001). Indeed, in response to the objection of some Orthodox Jews to the use of neurological criteria in diagnosing death, a 1987 New York regulation requires hospitals to have procedures for the "reasonable accommodation" of patients' religious or moral objections to the standards used to determine death (New York State Department of Health 2005). In 1991, New Jersey enacted a statute that has separate sections recognizing "traditional cardio-respiratory criteria" and "modern neurological criteria," and that prohibits the physician from using the latter when he or she "has reason to believe" that "a declaration [on the basis of neurological criteria] would violate the personal religious beliefs of the individual (New Jersey Office of the Attorney General 2007)." It is interesting to note that most of the discussion of organ donation stems from the focus on brain death or heart-beating donation. It is commonly assumed, without further formal discussion among religious leaders, that the justification of the more recent protocols of organ procurement in NHBOD, including in other states of impaired consciousness, can be derived from the agreement, on principle, that organ donation is a genuine act of beneficence. However, recent events and developments in organ procurement procedures have triggered a response from religious institutions. Scholars from diverse religious affiliations have revisited the opinions on brain death in light of contemporary medical knowledge (Brown 2007;
Diamond 2007; Kunin 2004; Shea 2007). The House of Lords European Union Committee published the 17th Report of Session 2007-2008 on Increasing the Supply of Donor Organs within the European Union (House of Lords European Union Committee 2008). The report recognizes that several major religious groups (as well as some individuals with no faith group affiliation) had major reservations about the concept of brain death and opposed organ donation from donors whose death has been defined solely on the basis of brain death. Several diverse religious groups oppose organ donation because of a fundamental belief that the human body is a trust that has been given and owned by God and, therefore, should not be physically violated by removing organs. Although Pope Pius XII declared that rigorously applying the criteria for ascertaining brain death suffices for arriving with moral certitude at the conclusion that death has occurred, Pope Benedict XVI has asked that the debate on brain death and organ procurement be revived (Shea 2007; Timesonline 2008). Pope Benedict XVI stated that vital organs can be extracted "ex cadavere" [from a dead body], if and only if, the donor's true death can be certified beyond a doubt (Pope Benedict XVI 2008). If the medical assumption of the moral certitude of brain death criteria turns out to be wrong, then we would no longer be able to use brain-death criteria with moral certainty (Brown 2007). In addition, it must be noted that, in 1957, a group of anesthesiologists posed the ethical problem of medical prolongation for life to Pope Pius XII and asked for instruction (Giacomini 1997). In 1958, Pope Pius XII referred the dilemma back to the doctors, affirming that the criteria for timing (not defining) death under artificial life support should be left to the attending physician. It is important to point out that, at that time, neither the press nor physicians interpreted the Pope's statement as a call to redefine death itself (Giacomini 1997). It is also widely recognized that the degree of certitude required in determining death is influenced by the anticipated removal of organs for transplantation (Diamond 2007). Shewmon argued that the medical community has fallen into the logical fallacy of accepting that the absence of evidence of conscious activity constitutes evidence of its absence (Shewmon 1997). Diamond concluded that the debate about brain death should be elevated to a truly scientific dialog about the significance of certain irreversible losses of function (Diamond 2007). It is therefore premature to conclude that no religious opposition exists to organ procurement procedures. We speculate that religious leaders will reopen the discussion on these issues in the near future.

Studies have shown that patients consider the most important end-of-life decision to be their wish to not be kept alive on life support when there is little hope for a meaningful recovery (Ivan and with contributions by Melrose 2007). If the clinical guidelines used in medical 
practice and accepted as medical standards for the determination of either brain death or other states of impaired consciousness cannot exclude catastrophic diagnostic errors or uncertainty about human death, then heart-beating organ donation and NHBOD are effectively physicianassisted death. We agree with Truog's analysis that formulation of the Harvard criteria for brain death and organ donation in 1968 marked the beginning of medical experimentation with physician-assisted death in the United States (Truog 2008). A concept of brain death may meet the criteria of a necessary condition for death but fails as a sufficient condition for a comprehensive understanding of death. The President's Council on Bioethics recognizes and acknowledges that there is no clinical or scientific evidence proving that death based on only neurological criteria thus indeed fully encompasses the concept of human death (The President's Council on Bioethics 2008, pp. 54-57). The Council reappraises the reality that the neurological standard of "whole brain death" corresponds to a "condition of profound incapacity, diagnostically distinct from all other cases of severe injury" (p. 38). Although the Council does not state it in so many words, it implies that over the past 40 years, all statutory death laws, all diagnostic criteria for "brain death," and all transplantations from heartbeating donors have, in retrospect, been based on an invalid conceptual framework and incorrect empirical facts. The validity of the Council's new philosophical rationale for continued justification of the concept of brain death has already been questioned (Shewmon 2009). Because of this, significant changes would be required in how we think about death and dying, how we provide and withdraw medical care from brain-dead patients and those in other states of impaired consciousness, and how we make decisions about the ethical permissibility of NHBOD. We have also previously argued that applying circulatory criterion for determining irreversible cessation of circulation and respiration (of the brain stem) in compliance with the UDDA, is not compatible with recovering transplantable organs in NHBOD (Verheijde et al. 2007a).

What are the practical implications for bedside clinicians? From medical and ethical perspectives, surgically procuring organs without general anesthesia, while failing to recognize that donors may not be really dead, can inflict unnecessary harm at the end of life. Harm includes the possibility of active inner awareness as well as the experience of pain and other primary-process affects in incipiently dying donors during surgical procurement (Giacino 1997). Death by organ procurement may also violate deeply rooted personal end-of-life values and beliefs of some donors. Procuring organs based on unsubstantiated criteria of death also raises legal questions about the compliance with homicide statutes (McGregor et al. 2008). Consenting to organ donation after death cannot be construed as consenting for physician-assisted death in order to procure transplantable organs. Donors or surrogates cannot consent to their own death unless such actions have already been legalized in society. For these reasons and in order to continue the current practice of organ procurement, we posited that recovery of transplantable organs from decedents requires a paradigm change in the ethics of organ donation (Verheijde et al. 2007a). Within the revised paradigm, the uncertainties about clinical determination of states of impaired consciousness and death in human beings, which also include brain death, are disclosed and discussed publically to maintain trust in the integrity of the medical profession. Furthermore, if the general public and society judge that the current degree of clinical uncertainty is acceptable, then establishing a legal definition of end-of-life care that would include physician-assisted death can be an option to resolve the existing conflicts in procuring transplantable organs from patients who have little hope for a meaningful recovery and who may be in states of impaired consciousness (Ivan and with contributions by Melrose 2007).

\section{Conclusions}

Brain death and vegetative state are two clinical conditions within a spectrum of pathologic states of impaired consciousness. The clinical accuracy of diagnosis and discrimination among different pathologic states of impaired consciousness remain open to scientific questioning and debate. The neurological standard to determine brain death fails to provide conclusive evidence that the brain-based criteria fulfill the concept of death because this state does not disrupt somatic integrative unity or coordinated biological functioning of a living organism. The determination of death in heart-beating donors by relying on brain-based criteria excludes relevant anthropologic, psychosocial, cultural, and religious aspects of death and dying. Current clinical guidelines for determining brain death do not require the confirmatory presence of structural disruption and ischemic injury of the brain stem on autopsy; therefore, the guidelines do not completely exclude reversible loss of integrated neurological functions. Wide practice variation and lack of compliance with the clinical guidelines for determining brain death before organ donation are generating concerns for potential misdiagnosis and incorrect declaration of brain death. The lack of compliance, scientific robustness, and discriminatory power of the clinical guidelines, which are accepted as a medical standard, increase the risk of physicians erring by determining reversible states of impaired consciousness as an irreversible state of brain death.

Brain-based criteria of death fail in determining the precise moment of an organism's death when death is declared in NHBOD protocols by circulatory criterion in 
patients with states of impaired consciousness and who cannot be declared clinically brain-dead. From a medical practice point of view, the definition of brain death confirms that the practice of NHBOD can only be justified if circulatory arrest is observed for longer than $15 \mathrm{~min}$ to allow for the onset of whole brain (including brain stem) ischemia and demonstrating irreversible cessation of integrated neurological functions and respiratory drive. It is unlikely that a waiting time of $75 \mathrm{~s}$ to $5 \mathrm{~min}$ is an appropriate amount of time for the whole brain to irreversibly cease functioning. As a result, the term brain death cannot be applied to validate and justify current practices in NHBOD in most medical institutions in the United States and elsewhere. Neuroimaging and electrophysiology studies of the vegetative state call into question the validity of clinical determination of this state of impaired consciousness. The scientific uncertainty of the definition and clinical imprecision of the determination of states of impaired consciousness (including brain death) have not been disclosed to the general public nor have they been broadly debated by the medical community or religious scholars. Heart-beating or non-heart-beating organ procurement from patients with impaired consciousness is in reality a concealed practice of physician-assisted death, and therefore violates both the criminal laws and central tenet of medicine of do-no-harm principle. Society must decide if physician-assisted death is permissible and desirable to resolve the conflict about procuring organs from patients with impaired consciousness and the perceived need to enhance the supply of transplantable organs.

Open Access This article is distributed under the terms of the Creative Commons Attribution Noncommercial License which permits any noncommercial use, distribution, and reproduction in any medium, provided the original author(s) and source are credited.

\section{References}

Adhiyaman, V., S. Adhiyaman, and R. Sundaram. 2007. The Lazarus phenomenon. Journal of the Royal Society of Medicine 100 (12): $552-557$.

Andrews, K., L. Murphy, R. Munday, and C. Littlewood. 1996. Misdiagnosis of the vegetative state: Retrospective study in a rehabilitation unit. British Medical Journal 313 (7048): 13-16.

Beecher, H., and Ad Hoc Committee of the Harvard Medical School to Examine the Definition of Brain-Death. 1968. A definition of irreversible coma. Special communication: Report of the Ad Hoc Committee of the Harvard Medical School to Examine the Definition of Brain Death. The Journal of the American Medical Association 205 (6): 337-340.

Boucek, M., C. Mashburn, S. Dunn, R. Frizell, L. Edwards, B. Pietra, et al. 2008. Pediatric heart transplantation after declaration of cardiocirculatory death. New England Journal of Medicine 359 (7): 709-714.

Brown, G. 2007. Reading the signs of death: A theological analysis. The National Catholic Bioethics Quarterly 7 (3): 467-476.
Byrne, P., and W. Weaver. 2004. "Brain death" is not death. Advances in Experimental Medicine \& Biology 550: 43-49.

Capron, A.M. 2001. Brain death-well settled yet still unresolved. The New England Journal of Medicine 344 (16): 1244-1246.

Committee on Increasing Rates of Organ Donation-Board on Health Sciences Policy-Institute of Medicine. 2006. Organ donation: Opportunities for action. Washington, DC: The National Academies Press.

Committee on Non-Heart-Beating Transplantation II-The Scientific, Ethical Basis for Practice and Protocols-Division of Health Care Services-Institute of Medicine. 2000. Non-heart-beating organ transplantation: Practice and protocols. Washington, DC: National Academy Press.

Council on Scientific Affairs, Council on Ethical, Judicial Affairs of the American Medical Association. 1990. Persistent vegetative state and the decision to withdraw or withhold life support. Council on Scientific Affairs and Council on Ethical and Judicial Affairs. The Journal of the American Medical Association 263 (3): 426-430.

Cusack, D., A. Sheikh, and J. Hyslop-Westrup. 2000. 'Near PVS': A new medico-legal syndrome? Medicine, Science and the Law 40 (2): $133-142$.

Dejohn, C., and J.B. Zwischenberger. 2006. Ethical implications of extracorporeal interval support for organ retrieval (EISOR). ASAIO (American Society for Artificial Internal Organs) Journal 52 (2): 119-122.

Detry, O., S. Laureys, M. Faymonville, A. De Roover, J. Squifflet, M. Lamy, et al. 2008. Organ donation after physician-assisted death. Transplant International 21 (9): 915.

DeVita, M.A., and J.V. Snyder. 1993. Development of the University of Pittsburgh Medical Center policy for the care of terminally ill patients who may become organ donors after death following the removal of life support. Kennedy Institute of Ethics Journal 3 (2): 131-143.

Diamond, E.F. 2007. John Paul II and brain death. The National Catholic Bioethics Quarterly 7 (3): 491-498.

Giacino, J.T. 1997. Disorders of consciousness: Differential diagnosis and neuropathologic features. Seminars in Neurology 17 (2): $105-111$

Giacomini, M. 1997. A change of heart and a change of mind? Technology and the redefinition of death in 1968. Social Science \& Medicine 44 (10): 1465-1482.

Greer, D.M., P.N. Varelas, S. Haque, and E.F.M. Wijdicks. 2008a. Variability of brain death determination guidelines in leading US neurologic institutions. Neurology 70: 284-289.

Greer, D.M., P.N. Varelas, S. Haque, and E.F.M. Wijdicks. 2008b. Variability of brain death determination guidelines in leading US neurologic institutions. Reply from authors. Neurology 71 (14): 1126.

Hoffenberg, R., M. Lock, N. Tilney, C. Casabona, A.S. Daar, R.D. Guttmann, et al. 1997. Should organs from patients in permanent vegetative state be used for transplantation? The Lancet 350 (9087): 1320-1321.

House of Lords European Union Committee. 2008. 17th report of session 2007-08. Increasing the supply of donor organs within the European Union volume I: Report. (HL Paper 123-I). Published 2 July 2008, from http://www.publications.parliament. uk/pa/ld200708/ldselect/ldeucom/123/123i.pdf.

Howard M. Nathan-Medscape Transplantation. 2005. The growing use of donation after cardiac death donors: An expert interview with Howard M. Nathan. Available at California Transplant Donor Network from www.ctdn.org/downloads/From_Medscape Transplantation.pdf.

Ivan, L., and with contributions by Melrose, M. 2007. The way we die. Brain death, vegetative state, euthanasia and other end-of-life dilemmas. Grosseto, Italy: Pari Publishing, Sas. 
Joffe, A.R. 2007a. The ethics of donation and transplantation: Are definitions of death being distorted for organ transplantation? Philosophy, Ethics, and Humanities in Medicine 2 (1): 18. Retrieved from http://www.peh-med.com/content/2/1/28.

Joffe, A.R. 2007b. The neurological determination of death: What does it really mean? Issues in Law and Medicine 23 (2): 119-140.

Joffe, A.R. 2009. Are recent defences of the brain death concept adequate? Bioethics, Early Online. doi:10.1111/j.1467-8519. 2008.00709.x.

Joffe, A.R., N. Anton, and V. Mehta. 2007. A survey to determine the understanding of the conceptual basis and diagnostic tests used for brain death by neurosurgeons in Canada. Neurosurgery 61 (5): 1039-1047.

Karakatsanis, K.G. 2008. Brain death: Should it be reconsidered? Spinal Cord 46 (6): 396-401.

Karakatsanis, K.G., and J.N. Tsanakas. 2002. A critique on the concept of 'brain death'. Issues in Law \& Medicine 18 (2): 127141.

Keep, P.J. 2000. Anaesthesia for organ donation in the brainstem dead. Anaesthesia 55 (6): 590.

Kellehear, A. 2008. Dying as a social relationship: A sociological review of debates on the determination of death. Social Science \& Medicine 66 (7): 1533-1544.

Kunin, J. 2004. Brain death: Revisiting the rabbinic opinions in light of current medical knowledge. Tradition 38 (4): 48-62.

Laureys, S., and J.J. Fins. 2008. Are we equal in death? Avoiding diagnostic error in brain death. Neurology 70 (4): e14-e15.

Lifesitenews.com. 2008. Woman's waking after brain death raises many questions about organ donation. http://www.lifesitenews. com/ldn/2008/may/08052709.html.

Long, T., M. Sque, and J. Addington-Hall. 2008. Conflict rationalisation: How family members cope with a diagnosis of brain stem death. Social Science \& Medicine 67 (2): 253-261.

Lynn, J. 1993. Are the patients who become organ donors under the Pittsburgh protocol for "non-heart beating donors" really dead? Kennedy Institute of Ethics Journal 3: 167-178.

Maruya, J., K. Nishimaki, J.-I. Nakahata, H. Suzuki, Y. Fujita, and T. Minakawa. 2008. Prolonged somatic survival of clinically braindead adult patient-case report. Neurologia Medico-Chirurgica 48 (3): 114-117.

Mathur, M., L. Petersen, M. Stadtler, C. Rose, J.C. Ejike, F. Petersen, et al. 2008. Variability in pediatric brain death determination and documentation in southern California. Pediatrics 121 (5): 988993.

McGregor, J.L., J.L. Verheijde, and M.Y. Rady. 2008. Do donation after cardiac death protocols violate criminal homicide statutes? Medicine and Law 27 (2): 241-257.

Morales, N. 2008. 'Dead' man recovering after ATV accident. Doctors said he was dead, and a transplant team was ready to take his organs-until a young man came back to life. Dateline NBC News. March 23, 2008, from http://www.msnbc.msn.com/ id $/ 23768436 /$.

National Conference of Commissioners on Uniform State Laws. 1981. The Uniform Determination of Death Act 1981, from http://www.law.upenn.edu/bll/ulc/fnact99/1980s/udda80.htm.

New Jersey Office of the Attorney General. 2007. New Jersey register volume 39, issue 9, issue date: May 7, 2007. Rule adoptions law and public safety division of consumer affairs board of medical examiners. Adopted repeal and new rules: N.J.A.C. 13:35-6A. Declarations of death upon the basis of neurological criteria, from http://www.state.nj.us/oag/ca/adoption/bmeado57.htm.

New York State Department of Health. 2005. New York State Department of Health Guidelines for Determining Brain death, from http://www.health.state.ny.us/professionals/doctors/guide lines/determination_of_brain_death/docs/ determination_of_brain_death.pdf.
Owen, A.M., and M.R. Coleman. 2008. Functional neuroimaging of the vegetative state. Nature Reviews Neuroscience 9 (3): 235243.

Panksepp, J., T. Fuchs, V.A. Garcia, and A. Lesiak. 2007. Does any aspect of mind survive brain damage that typically leads to a persistent vegetative state? Ethical considerations. Philosophy, Ethics, and Humanities in Medicine 2 (1): 32. Retrieved from http://www.peh-med.com/content/2/1/32/.

Paradis, N.A., G.B. Martin, E.P. Rivers, M.G. Goetting, T.J. Appleton, M. Feingold, et al. 1990. Coronary perfusion pressure and the return of spontaneous circulation in human cardiopulmonary resuscitation. The Journal of the American Medical Association 263 (8): 1106-1113.

Pope Benedict XVI. 2008. Benedict XVI on organ donation. November 7, 2008, from http://www.zenit.org/article-24191?]= english.

President's Commission for the Study of Ethical Problems in Medicine and Biomedical and Behavioral Research. 1981. Defining death: A report on the medical, legal and ethical issues in the determination of death, from http://www.bioethics.gov/ reports/past_commissions/index.html.

Quill, T.E. 2007. Legal regulation of physician-assisted death-the latest report cards. The New England Journal of Medicine 356 (19): 1911-1913.

Rady, M., J. Verheijde, and J.L. McGregor. 2007. Autoresuscitation and organ donation after cardiac death: Clarifying misunderstandings about the physiology of human circulation. Philosophy, Ethics, and Humanities in Medicine, from http://www.pehmed.com/content/2/1/18/comments\#285644.

Report of the Quality Standards Subcommittee of the American Academy of Neurology. 1995. Practice parameters: Assessment and management of patients in the persistent vegetative state (summary statement). Neurology 45: 1015-1018.

Rifkinson-Mann, S. 2003. Legal consequences and ethical dilemmas of pain perception in persistent vegetative states. Journal of Health Law 36 (4): 523-548.

Shea, J.B. 2007. Organ donation: The inconvenient truth. Catholic Insight,

from http://catholicinsight.com/online/bioethics/article_747.shtml.

Shemie, S.D. 2007. Clarifying the paradigm for the ethics of donation and transplantation: Was 'dead' really so clear before organ donation? Philosophy, Ethics, and Humanities in Medicine 2 (1): 18. Retrieved from http://www.peh-med.com/content/2/1/18.

Shewmon, D.A. 1997. Recovery from 'brain death:' A neurologist's apologia. Linacre $Q$ 64: 30-85.

Shewmon, D.A. 1998. Chronic "brain death": Meta-analysis and conceptual consequences. Neurology 51 (6): 1538-1545.

Shewmon, D.A. 2001. The brain and somatic integration: Insights into the standard biological rationale for equating 'brain death' with death. Journal of Medicine \& Philosophy 26 (5): 457-478.

Shewmon, D.A. 2004. A critical analysis of conceptual domains of the vegetative state: Sorting fact from fancy. Neuro Rehabilitation 19 (4): 343-347.

Shewmon, D.A. 2009. Brain death: Can it be resuscitated? Hastings Center Report 39 (2): 18-23.

The House of Representatives of the 144th General Assembly of the State of Delaware. 2008. House resolution no. 75. Establishing protections for persons with severe brain injury, impaired consciousness, or who are otherwise mentally disabled, from http://legis.delaware.gov/LIS/lis 144.nsf/vwLegislation/HR+75/ \$file/legis.html.

The New England Journal of Medicine (Online). 2008. Perspective roundtable: Organ donation after cardiac death, from http://content.nejm.org/cgi/content/full/359/7/669/DC1.

The President's Council on Bioethics. 2007a. Session 1: The draft white paper on the determination of death. Discussion by council 
members, from http://www.bioethics.gov/transcripts/sept07/ session 1.html.

The President's Council on Bioethics. 2007b. Session 5: Response to the council's white paper, "controversies in the determination of death" (D. Alan Shewmon), from http://www.bioethics.gov/ transcripts/nov07/session5.html.

The President's Council on Bioethics. 2008. Controversies in the determination of death. A white paper of the President's Council on Bioethics. December 2008. www.bioethics.gov Washington, DC, from http://www.bioethics.gov/reports/.

The Quality Standards Subcommittee of the American Academy of Neurology. 1995. Practice parameters for determining brain death in adults (summary statement). Neurology 45 (5): 1012 1014.

Timesonline. 2008. Owen Richard (Rome). Vatican called on to reopen debate on brain death as end of life. From Times Online, September 3, 2008, from http://www.timesonline.co.uk/tol/ comment/faith/article4667507.ece.

Truog, R. 2007. Brain death-too flawed to endure, too ingrained to abandon. Journal of Law Medicine \& Ethics 35 (2): 273-281.

Truog, R. 2008. End-of-life decision-making in the United States. European Journal of Anaesthesiology 25 (Supplement S42): 4350.

Truog, R., and F. Miller. 2008. The dead donor rule and organ transplantation. The New England Journal of Medicine 359 (7): 674-675.

Verheijde, J.L., M.Y. Rady, and J.L. McGregor. 2007a. Recovery of transplantable organs after cardiac or circulatory death: Transforming the paradigm for the ethics of organ donation. Philosophy, Ethics, and Humanities in Medicine 2 (1): 8. Retrieved from http://www.peh-med.com/content/2/1/8.
Verheijde, J.L., M.Y. Rady, and J.L. McGregor. 2007b. The United States Revised Uniform Anatomical Gift Act (2006): New challenges to balancing patient rights and physician responsibilities. Philosophy, Ethics, and Humanities in Medicine 2 (1): 19. Retrieved from http://www.peh-med.com/content/2/1/19.

Walker, A.E. 1978. Pathology of brain death. Annals of the New York Academy of Sciences 315: 272-280.

Wijdicks, E.F. 2001. The diagnosis of brain death. The New England Journal of Medicine 344 (16): 1215-1221.

Wijdicks, E.F., and R.E. Cranford. 2005. Clinical diagnosis of prolonged states of impaired consciousness in adults. Mayo Clinic Proceedings 80 (8): 1037-1046.

Wijdicks, E.F., A. Hijdra, G.B. Young, C.L. Bassetti, and S. Wiebe. 2006. Practice parameter: Prediction of outcome in comatose survivors after cardiopulmonary resuscitation (an evidencebased review): Report of the Quality Standards Subcommittee of the American Academy of Neurology. Neurology 67 (2): 203210.

Wijdicks, E.F., and E.A. Pfeifer. 2008. Neuropathology of brain death in the modern transplant era. Neurology 70 (15): 1234-1237.

Woien, S., M.Y. Rady, J.L. Verheijde, and J.L. McGregor. 2006. Organ procurement organizations internet enrollment for organ donation: Abandoning informed consent. BMC Medical Ethics 7 (12): 14-23. Retrieved from http://www.biomedcentral.com/ $1472-6939 / 7 / 14$.

Young, P.J., and B.F. Matta. 2000. Anaesthesia for organ donation in the brainstem dead-why bother? Anaesthesia 55 (2): 105-106.

Zamperetti, N., R. Bellomo, C.A. Defanti, and N. Latronico. 2004. Irreversible apnoeic coma 35 years later. Intensive Care Medicine 30 (9): 1715-1722.

Zeman, A. 2001. Consciousness. Brain 124 (7): 1263-1289. 